\title{
Dietary fatty acids and risk of hepatocellular carcinoma in the Singapore Chinese Health Study
}

\author{
Woon-Puay Koh ${ }^{1,2}$, Yock Young Dan ${ }^{3,4}$, George Boon-Bee Goh ${ }^{1,5}$, Aizhen Jin $^{6}$, Renwei \\ Wang $^{7}$, and Jian-Min Yuan ${ }^{7,8}$ \\ ${ }^{1}$ Duke-NUS Graduate Medical School, National University of Singapore, Singapore 169857 \\ ${ }^{2}$ Saw Swee Hock School of Public Health, National University of Singapore, Singapore 117549 \\ ${ }^{3}$ University Medicine Cluster, Division of Gastroenterology, National University Health System, \\ Singapore 119228 \\ ${ }^{4}$ Department of Medicine, Yong Loo Lin School of Medicine, National University of Singapore and \\ National University Health System, Singapore 119228 \\ ${ }^{5}$ Department of Gastroenterology \& Hepatology, Singapore General Hospital, Singapore 169608 \\ ${ }^{6}$ National Registry of Diseases Office, Health Promotion Board, Singapore 168937 \\ ${ }^{7}$ Division of Cancer Control and Population Sciences, University of Pittsburgh Cancer Institute, \\ Pennsylvania, PA 15232 USA \\ ${ }^{8}$ Department of Epidemiology, University of Pittsburgh Graduate School of Public Health, \\ Pittsburgh, Pennsylvania, PA 15261, USA
}

\section{Abstract}

Background \& Aim-Lipidomic signature of lipid metabolism suggests that omega-6 polyunsaturated fatty acids (PUFA) may play a role in oncogenesis of hepatocellular carcinoma (HCC). Hence, we examined the association between dietary fatty acids and risk of HCC.

Methods-We used data from the Singapore Chinese Health Study, a population-based prospective cohort of 63,257 Chinese men and women aged 45-74 years enrolled between 1993 and 1998. Information on current diet assessed via a validated semi-quantitative food frequency questionnaire, medical history and lifestyle factors were obtained through in-person interview, and incidence of HCC recorded through 31 Dec 2010. We also examined the association between dietary fatty acids and HCC risk using a case-control set of 92 cases and 274 controls with available serological biomarkers of chronic infections with hepatitis B virus (HBV) and hepatitis C virus (HCV) nested within this cohort.

Results-Among the dietary fat components examined, which included saturated, monounsaturated, omega-3 and omega-6 PUFA, only omega-6 PUFA intake displayed a dosedependent, positive association with HCC risk ( $\mathrm{p}$ for trend=0.02). Compared to the lowest quartile,

Correspondence to: Dr Woon-Puay Koh, Office of Clinical Sciences, Duke-NUS Graduate Medical School Singapore, 8 College Road Level 4, Singapore 169857, Singapore. Phone: (65) 6601 3147; Fax: (65) 6222 7453; woonpuay.koh@ duke-nus.edu.sg. 
the hazard ratio for the highest quartile intake was 1.49 [(95\% confidence interval (CI): 1.08-2.07)]. In the nested case-control study, only among individuals negative for serology markers of chronic infection with $\mathrm{HBV}$ or $\mathrm{HCV}$, those who consumed above median levels of dietary omega-6 PUFA had increased HCC risk (odds ratio $=4.36,95 \% \mathrm{CI}=1.59-11.94$ ) compared to those with lower intake.

Conclusion-Dietary omega-6 PUFA may be implicated in the risk of non-viral hepatitis related HCC.

\section{Keywords}

hepatocellular carcinoma; dietary fatty acids; polyunsaturated fatty acids; omega- 6 fatty acids; non-alcoholic steatohepatitis

\section{Introduction}

Several recent studies have reported a correlation between specific diets and risk of developing hepatocellular carcinoma (HCC) (1-3). This is not unexpected since liver is the organ that metabolizes most of the nutrients and other dietary compounds absorbed from the gastrointestinal tract. A high intake of red meat and saturated fat has been associated with increased risk of HCC in the National Institutes of Health-AARP Diet and Health Study cohort in USA (4). Conversely, the European Prospective Investigation into Cancer and Nutrition (EPIC) cohort did not find any association with saturated fat, but reported an inverse association with monounsaturated fat instead (5). In other studies, consumption of fish, a rich source of omega-3 polyunsaturated fatty acids (PUFA), was associated with reduced risk of $\mathrm{HCC}(6,7)$. Furthermore, aspirin use was associated with a statistically significant $41 \%$ decrease in the risk of HCC among the National Institutes of Health-AARP Diet and Health Study study population (8). Aspirin can irreversibly inactivate cyclooxygenase (COX), the enzyme required for the conversion of arachidonic acid, an omega-6 PUFA derived from other dietary omega-6 PUFA which include gamma-linolenic and linoleic acids, to prostaglandins that have been implicated in chronic inflammation underlying carcinogenesis $(9,10)$. Recent work on the lipidomic signature of lipid metabolism also suggests that long chain omega-6 PUFA may play a role in hepatocarcinogenesis (11). However, little is known about the relationship between consumption of dietary omega-6 PUFA and risk of HCC in human population. Hence, we examined the association between dietary total fat and its major components including saturated fats, monounsaturated fats, omega- 3 and omega-6 PUFA and risk of HCC in a population-based prospective cohort of more than 60,000 middle-aged and older Chinese men and women in Singapore.

\section{Materials and Methods}

\section{Study Population}

The Singapore Chinese Health Study is a population-based prospective cohort established between April 1993 and December 1998 by enrolling 63,257 Chinese men $(n=27,959)$ and women $(n=35,298)$ aged $45-74$ years and who were residing in public housing estates, where about $86 \%$ of Singapore resided at that time of recruitment (12). The cohort study recruited 
only participants belonging to one of the two major Chinese dialect groups in Singapore, the Hokkiens or the Cantonese, who originated from two contiguous prefectures in southern China. The study was approved by the Institutional Review Board of the National University of Singapore, and all enrolled subjects gave informed consent.

We excluded individuals who had history of invasive cancer at baseline (except nonmelanoma skin cancer) either by self-report or via record linkage analysis with the nationwide Singapore Cancer Registry $(\mathrm{n}=1,936)$, as well as those who reported extreme sex-specific energy intakes ( $<600$ or $>3000 \mathrm{kcal} / \mathrm{d}$ for women and $<700$ or $>3700 \mathrm{kcal} / \mathrm{d}$ for men; $\mathrm{n}=1,023)$. The current analysis included 60,298 participants.

\section{Assessment of Diet and Covariates}

At recruitment, each subject was interviewed at home by a trained interviewer using a structured questionnaire, which covered demographics, lifetime use of tobacco, alcohol consumption, current physical activity, menstrual/reproductive history (for women), occupational exposure, medical history and family history of cancer. A semi-quantitative food-frequency questionnaire including 165 commonly consumed food items in this population was administered during the baseline interview. The respondents were instructed to select from 8 food-frequency categories (ranged from "never or hardly ever" to "two or more times a day") and three portion sizes (small, median, large) with the aid of photographs. The dietary intake of each nutrient was derived from the Singapore Food Composition Database, which has been described in detail previously (12). The foodfrequency questionnaire had been validated subsequently using 24-hour recalls and readministration of the food-frequency questionnaire among a subset of 810 participants from this cohort (12).

In this cohort, the main dietary sources of marine omega-3 PUFA, such as eicosapentaenoic acid and docosahexaenoic acid, were fish and seafood, while the main dietary sources of nonmarine omega-3 PUFA, such as alpha-linolenic acid (ALA), were grains (21\% of omega-3 PUFA intake), cooking oils (11\%), and legumes and soy (9\%). The main dietary sources of omega-6 PUFA were cooking fat/oil (40\%), grain products (20\%) and legumes $(7 \%)$.

\section{Case ascertainment}

We identified incident cases of cancers and deaths among cohort members through the record linkage analysis of the cohort database with databases of the population-based Singapore Cancer Registry and Singapore Registry of Births and Deaths. As of December 31,2010 , only 47 cohort subjects $(0.07 \%)$ were lost to follow-up due to migration out of Singapore or other reasons. The nationwide cancer registry has been in place since 1968 and has been shown to be comprehensive in its recording of incident cancer cases (13). In the cancer registry, only primary liver cancers were included, and among those with histological diagnosis, the vast majority of primary liver cancer cases were HCC. 


\section{Hepatitis B and C Serology}

Between April 1994 and December 1999, we collected blood and single-void urine specimens from a random 3\% sample of study enrollees. Starting in January 2000, the biospecimen collection was extended to all surviving cohort members. By April 2005 when all subjects had been contacted, biospecimens were collected from 32,543 participants, representing a roughly $60 \%$ consent rate.

We had previously constructed a case-control study nested within the Singapore Chinese Health Study; cases were all subjects who developed HCC after they donated a blood sample (14). For each case, three control subjects were randomly chosen among all eligible cohort participants who were alive and free of any cancer at the time of cancer diagnosis of their respective index case. The control subjects were individually matched to their index cases on year at recruitment ( \pm 1 year), year of birth ( \pm 2 years), gender, dialect group (Cantonese, Hokkien), and date of biospecimen collection ( \pm 6 months). For 92 HCC cases and 274 individually matched control subjects, we tested for the presence of hepatitis B surface antigen (HBsAg), antibodies to hepatitis B core antigen (anti-HBc) and antibodies to hepatitis B surface antigen (anti-HBs) and antibodies to hepatitis C virus (anti-HCV) as previously described (14).

\section{Statistical Analysis}

Person-years for each participant were calculated from the date of recruitment to date of HCC diagnosis, migration out of Singapore, death or December 31, 2010, whichever came first. Nutrient density intake of each fat component for individual participants is computed by dividing the intake of the fat component by total energy intake and expressing that in percentage of calories (\% kcal). Quartiles or median of density intake for each nutrient were based on the combined distribution of men and women in the whole cohort. Chi-square test for categorical variables and one-way ANOVA or Student's t-test for continuous variables were used to compare baseline characteristics among the different quartile intake categories of omega-6 PUFA and between the cases of incident HCC and the non-cases in the cohort.

The Cox proportional hazards regression method was used to examine the association between intake of dietary fatty acids and risk of HCC. We used the multivariate nutrient density model in which nutrient intake is expressed as percentage of calories, and total energy intake is added as a covariate in the model to address the potential problem of confounding (15). All models also included the following covariates measured at baseline: age at recruitment (years), gender, year of cohort enrollment (1993-1995, 1996-1998), dialect group (Hokkien, Cantonese), body mass index (BMI) $(<20,20-<24,24-<28,28+$ $\mathrm{kg} / \mathrm{m}^{2}$ ), level of education (no formal schooling, primary school, secondary school or higher), cigarette smoking status (never, former, or current smoker), consumption of alcoholic beverages (none or monthly, weekly, daily), coffee consumption $(<1,1,2-3,4+$ cups/day) and self-reported history physician-diagnosed diabetes, total energy and dietary intake of protein (quartiles). Additional analysis included adjustment for diet saturated fat, monounsaturated fat, omega-3 PUFA and omega-6 PUFA (all in quartiles). Linear relationship between dietary fatty acids and $\mathrm{HCC}$ risk were examined by entering ordinal values of the quartiles as a continuous variable in the model. We also explored the 
interaction between omega-6 PUFA intake and history of diabetes, BMI categories and alcohol consumption. The heterogeneity of the associations by different factors was tested using an interaction term (product between omega-6 PUFA intake categories and factor of interest) in the Cox model.

Within the nested case-control set of cohort subjects, we examined the association between dietary fatty acids and HCC risk stratified by subjects' status on viral serology using the unconditional logistic regression methods. For sensitivity analysis, we repeated our analyses with all the nutrients, including omega-6 PUFA, being adjusted for energy using the residual method (15).

We used SAS statistical software version 9.2 (SAS Institute, Cary, NC). All $P$ values quoted are two sided. $P$ values less than 0.05 were considered statistically significant.

\section{Results}

As of 31 December 2010, we identified 488 subjects who developed HCC after an average of 14.0 [standard deviation (SD) 3.7] years of follow-up. The mean age $( \pm \mathrm{SD})$ at diagnosis of HCC for cases was $69.2( \pm 8.1)$ years. The mean time interval $( \pm \mathrm{SD})$ between baseline interview and diagnosis of HCC was 8.3 (4.5) years. Compared to those with lesser intake, participants in the highest quartile of omega-6 PUFA intake were slightly younger and more likely to be women. They were also more likely to attain higher level of education, and were less likely to have smoked cigarettes, consumed alcoholic beverage at least weekly or drunk at least 2 cups of coffee daily. However, they had a higher prevalence of diabetes at recruitment and consumed higher levels of dietary protein and total fat (Table 1). Compared to the rest of the cohort, HCC cases were older at recruitment and more likely to be men. They were also less educated and more likely to be ever smokers or to consume alcohol at least weekly, but less likely to drink at least 2 cups of coffee per day. They also had a higher prevalence of diabetes at recruitment (Table 2).

Among all specific fatty acids including saturated fat, monounsaturated fat, omega-3 PUFA and omega-6 PUFA, as well as the omega-6 to omega-3 PUFA ratio, only omega-6 PUFA intake displayed a significant dose-dependent, positive association with HCC risk ( $P$ for trend $=0.02$ ). Compared to the lowest quartile, the HRs (95\% CIs) for quartiles 2, 3 and 4 were 1.27 (0.96-1.66), 1.39 (1.03-1.88) and 1.49 (1.08-2.07), respectively (Table 3). Although there was increased risk in the highest quartile compared to the lowest quartile intake for saturated fat, the HR (95\% CI) of $1.40(0.95-2.06)$ and the $P$ for trend $(0.20)$ did not reach statistical significance. Similarly, the reduced risk observed in quartile 4 compared to quartile 1 for intake for monounsaturated fat also did not reach statistical significance; HR $(95 \%$ CI) was $0.90(0.59-1.37), P$ for trend $=0.56$.

We did further analyses stratified by BMI categories $\left(<23 \mathrm{~kg} / \mathrm{m}^{2}\right.$ and $\left.23+\mathrm{kg} / \mathrm{m}^{2}\right)$ and history of diabetes, based on the cutoff value recommended for potential public health action points for Asian populations by the World Health Organization (WHO) (16). The association between intake of omega-6 PUFA and HCC risk was much stronger in those who were overweight/obese $\left(23+\mathrm{kg} / \mathrm{m}^{2}\right)$ compared to their leaner counterparts. Among the 
overweight/obese participants, omega-6 PUFA intake displayed a significant dosedependent, positive association with HCC risk ( $P$ for trend $=0.003)$; the HR $(95 \% \mathrm{CI})$ for the highest quartile was 1.91 (1.25-2.90). Comparatively, among the lean participants ( $<23 \mathrm{~kg}$ / $\mathrm{m}^{2}$ ), the association was null ( $P$ for trend=0.95); the HR $(95 \% \mathrm{CI})$ for the highest quartile was 0.96 (0.55-1.66) and $P$ for interaction between the two BMI categories and intake of omega-6 PUFA was 0.003 .

In the same way, the association between intake of omega-6 PUFA and HCC risk was stronger in those with baseline diabetes. Among these participants, omega-6 PUFA intake displayed a significant dose-dependent, positive association with HCC risk ( $P$ for trend=0.04); the HR (95\% CI) for the highest quartile was 1.88 (0.86-4.09). Comparatively, among those without diabetes, the association was weaker ( $P$ for trend $=0.10)$; the HR $(95 \%$ CI) for the highest quartile was $1.40(0.97-2.01)$ and $P$ for interaction between diabetes and intake of omega-6 PUFA was 0.15 .

Furthermore, we did analysis stratified by alcohol consumption. The association between intake of omega-6 PUFA and HCC risk was confined to non-drinkers, the HR $(95 \% \mathrm{CI})$ for the highest quartile of omega-6 PUFA was $1.74(1.2-2.51), P$ for trend=0.006. In contrast, among weekly/daily drinkers of alcohol, the association was null ( $P$ for trend $=0.86$ ); the HR (95\% CI) for the highest quartile was $1.01(0.46-2.20)$ and $P$ for interaction between the alcohol consumption and intake of omega-6 PUFA was 0.04 . Finally, we analysed the data among those with under 4 years of follow-up (short duration) and those with follow-up duration of 4 years or more (long duration), and the positive, dose-dependent associations between omega-6 PUFA intake and HCC risk were present and similar in both groups, although the results did not reach statistical significance due to smaller case numbers in the stratified analysis ( $\mathrm{P}$ for trend $=0.09$ for short duration vs 0.06 for long duration).

In the case-control set nested within this cohort that included 92 cases and 274 controls with available serological markers of hepatitis B and C infection, higher consumption of omega-6 PUFA (above median intake level) or higher omega-6 to omega-3 PUFA ratio (above median) was associated with statistically significant increase in risk of HCC overall (Table 4). When subjects were stratified by seropositivity of HBV and HCV infection, higher intake of omega-6 PUFA or higher omega- 6 to omega-3 PUFA ratio was positive associated with $\mathrm{HCC}$ risk only among individuals negative for $\mathrm{HBsAg}$, anti-HBc, and anti-HCV (38 cases and 221 controls); odds ratio of HCC for higher (above median) versus lower (below median) intake was 4.36 (95\% CI: 1.59-11.94) for omega-6 PUFA and 2.98 (95\% CI: 1.26-7.05) for the omega-6 to omega-3 PUFA ratio (Table 5). The interaction between seropositivity and omega-6 PUFA intake on HCC risk was of borderline statistical significance ( $P$ for interaction $=0.08$ ). We repeated our analyses by adjusting for energy using the residual method in a sensitivity analysis, and the results remained the same. Among individuals negative for $\mathrm{HBsAg}$, anti-HBc, and anti-HCV, odds ratio of $\mathrm{HCC}$ for higher (above median) versus lower (below median) intake was 4.01 (95\% CI: 1.41-11.40) for omega-6 PUFA and 2.32 (95\% CI: 0.93-5.80) for the omega-6 to omega-3 PUFA ratio. 


\section{Discussion}

In this large cohort study of Chinese men and women, intake of omega-6 PUFA was associated with increased risk of HCC. Although dietary omega-3 PUFA was not associated with overall risk of HCC, higher ratio of omega- 6 to omega-3 PUFA conferred an increased risk of HCC. These results provided the first epidemiologic evidence for the role of dietary omega-6 PUFA in the development of HCC that was presumably due to underlying nonalcoholic steatohepatitis (NASH).

Metabolic derivatives from omega-6 PUFA are thought to be pro-inflammatory while those from omega-3 PUFA have anti-inflammatory properties (17). Studies suggest that omega-3 PUFA improves insulin sensitivity and reduce inflammation in NASH via their action on circulating triglyceride levels and regulating gene transcription factors involved in hepatic lipid metabolism, fatty acid oxidation and inflammatory pathways $(18,19)$. Conversely, metabolism of omega-6 PUFA leads to increased levels of pro-inflammatory products, such as prostaglandin $\mathrm{E} 2$, thromboxane and indirectly $\mathrm{C}$-reative protein, plasminogen activator inhibitor (PAI)-1, TNF- $a$ and IL-6 (20, 21), which have been implicated in causing advanced fibrosis in NASH, and subsequently to cirrhosis and ultimately to $\mathrm{HCC}(22,23)$. NASH patients, compared to controls, were reported to have a significant increase of omega-6 PUFA levels within hepatic lipid content, while omega-3 PUFA levels were comparable (24). Similarly, when dietary patterns were assessed, dietary intake of omega-3 PUFA was comparable between NASH patients and controls, while dietary intake of omega-6 PUFA was significantly higher in NASH patients relative to controls (25). Lipidomic studies in phosphatase and tensin homolog (Pten) null mouse models with NASH demonstrated a positive correlation between omega-6 PUFA and HCC tumour burden, but a negative correlation between omega-3 PUFA and tumour burden (11).

Although we did not do full etiological characterization for all cases, local and international epidemiology data indicate that the majority of these non-viral hepatitis related HCC in our population are due to NASH (26). Furthermore, the Singapore Chinese Health Study is a cohort with a very low prevalence of frequent alcohol intake (27). Since we did not have serological markers of hepatitis B and C infection from all the cohort participants, we were unable to differentiate between viral-hepatitis related HCC and HCC that was presumably due to underlying NASH in analysis involving all cohort participants. However, as diabetes $(28,29)$ and overweight/obesity (30) are established factors of HCC related to NASH, we did stratified analyses, and our results showed that the positive association between omega- 6 PUFA intake and HCC risk was much stronger among those who were overweight/obese or among those with diabetes, and also seemed to be confined to nondrinkers of alcohol. Hence, we postulate that the influence of omega-6 PUFA on non-viral related HCC may be driven via its effects on the pathogenesis of NASH extending to cirrhosis and subsequent HCC (31). Since HCC related to chronic HBV/HCV infection is known to arise from different carcinogenic pathways $(32,33)$, it is not unexpected that the association between dietary omega-6 PUFA and HCC risk was not observed in HBV/HCV seropositive cases in our study. 
Since case-control studies, particularly those with hospital-based controls $(2,34,35)$, have inherent limitation such as recall bias, temporal bias and selection bias in the study design, for the purpose of comparison with our findings, we have focused on other population-based prospective studies. The US National Institutes of Health-AARP Diet and Health Study previously reported an increased risk of HCC with increased intake of red meat and saturated fat (4). In contrast, the large European Prospective Investigation into Cancer and Nutrition (EPIC) cohort did not report any association with saturated fat. Instead, they found an inverse association with monounsaturated fat intake (5). In this cohort of Chinese in Singapore, although we observed increased risk of HCC in the higher quartiles compared to the lowest quartile for saturated fat intake, and also decreased risk in the higher quartiles compared to the lowest quartile for monounsaturated fat intake, these associations did not reach statistical significance. We have previously reported that the intake of all components of dietary fat, including saturated fat, monounsaturated fat and polyunsaturated fat, were lower in this cohort of Singapore Chinese than among US population (12). Unlike the US or European populations, the main type of red meat consumed in this population was pork rather than beef or lamb (36). We postulate that these differences in intake levels and food sources of fat could account for the non-significant associations with saturated and monounsaturated fats in our study. Finally, our finding of an association between higher omega- 6 to omega-3 PUFA ratio and increased risk of HCC in our nested case-control study, especially for the non-viral hepatitis related HCC, was indirectly consistent with reports from the Japanese cohort and the EPIC cohort that fish or omega-3 PUFA intake could reduce $\mathrm{HCC}$ risk $(6,7)$.

The strengths of our study include the high response and follow-up rates, detailed collection of data through face-to-face interviews and a comprehensive capture of incident cancer cases using the nationwide cancer registry in a country such as Singapore, which has a system for easy access to specialized medical care and where notification of cancer is required by law. Another strength is the presumed lack of recall bias in the data on diet and other covariates since they were obtained prior to disease diagnosis. We included established and other possible risk factors for $\mathrm{HCC}$ as covariates in our regression-based risk models to minimize the likelihood of spurious associations resulting from insufficient control of confounding. The dietary intake was assessed by a food-frequency questionnaire that was specifically developed and validated in this population, and has been shown to be internally consistent and reproducible (12). Limitations of the study include the use of self-report for dietary intake, as some levels of measurement error were inevitable. However, this would most likely result in non-differential misclassification with respect to disease status and probable underestimation of the risk estimates. We also did not have periodic assessment of the diet to allow us to examine the potential reported changes in these factors in relation to the outcomes. Third, we did not collect any information about the history of chronic liver disease to determine the prevalence of fatty liver disease or NASH at recruitment. We also recognize that the null associations observed among the subjects positive for $\mathrm{HBV}$ or $\mathrm{HCV}$ could be explained by insufficient statistical power due to relatively small case numbers in this subgroup. Finally, due to the observational study nature, causality inference should be made with caution. 
In conclusion, this study reports the novel finding that high dietary intake of omega 6 PUFA may increase the risk of non-viral hepatitis related HCC. This association may be due to the effect of omega-6 PUFA on rendering chronic inflammation in NASH, which is an increasingly important cause of liver disease and liver cancer in the world. Future research in mechanistic and intervention studies is warranted to evaluate the effect of dietary fat in relation to HCC of different etiologies.

\section{Acknowledgments}

We thank Siew-Hong Low of the National University of Singapore for supervising the fieldwork in the Singapore Chinese Health Study. We also thank The Singapore Cancer Registry for the identification of cancer and mortality outcomes via database linkages. Finally, we acknowledge the founding Principal Investigator of the Singapore Chinese Health Study, Mimi C. Yu.

Funding: This study was supported by the National Institutes of Health, USA (RO1 CA144034 and UM1 CA182876).

\section{References}

1. Montella M, Crispo A, Giudice A. HCC, diet and metabolic factors: Diet and HCC. Hepat Mon. 2011; 11:159-62. [PubMed: 22087137]

2. Turati F, Trichopoulos D, Polesel J, Bravi F, Rossi M, Talamini R, et al. Mediterranean diet and hepatocellular carcinoma. J Hepatol. 2014; 60:606-11. [PubMed: 24240052]

3. Mandair DS, Rossi RE, Pericleous M, Whyand T, Caplin M. The impact of diet and nutrition in the prevention and progression of hepatocellular carcinoma. Expert Rev Gastroenterol Hepatol. 2014; 8:369-82. [PubMed: 24597926]

4. Freedman ND, Cross AJ, Mcglynn KA, Abnet CC, Park Y, Hollenbeck AR, et al. Association of meat and fat intake with liver disease and hepatocellular carcinoma in the NIH-AARP cohort. J Natl Cancer Inst. 2010; 102:1354-65. [PubMed: 20729477]

5. Duarte-Salles T, Fedirko V, Stepien M, Aleksandrova K, Bamia C, Lagiou P, et al. Dietary Fat, Fat Subtypes and Hepatocellular Carcinoma in a Large European Cohort. Int J Cancer. 2015

6. Sawada N, Inoue M, Iwasaki M, Sasazuki S, Shimazu T, Yamaji T, et al. Consumption of n-3 fatty acids and fish reduces risk of hepatocellular carcinoma. Gastroenterology. 2012; 142:1468-75. [PubMed: 22342990]

7. Fedirko V, Trichopolou A, Bamia C, Duarte-Salles T, Trepo E, Aleksandrova K, et al. Consumption of fish and meats and risk of hepatocellular carcinoma: the European Prospective Investigation into Cancer and Nutrition (EPIC). Ann Oncol. 2013; 24:2166-73. [PubMed: 23670094]

8. Sahasrabuddhe VV, Gunja MZ, Graubard BI, Trabert B, Schwartz LM, Park Y, et al. Nonsteroidal anti-inflammatory drug use, chronic liver disease, and hepatocellular carcinoma. J Natl Cancer Inst. 2012; 104:1808-14. [PubMed: 23197492]

9. Reddy BS, Maeura Y. Tumor promotion by dietary fat in azoxymethane-induced colon carcinogenesis in female F344 rats: influence of amount and source of dietary fat. J Natl Cancer Inst. 1984; 72:745-50. [PubMed: 6583457]

10. Dayton S, Hashimoto S, Wollman J. Effect of high-oleic and high-linoleic safflower oils on mammary tumors induced in rats by 7,12-dimethylbenz(alpha)anthracene. J Nutr. 1977; 107:135360. [PubMed: 196058]

11. Muir K, Hazim A, He Y, Peyressatre M, Kim DY, Song X, et al. Proteomic and lipidomic signatures of lipid metabolism in NASH-associated hepatocellular carcinoma. Cancer Res. 2013; 73:4722-31. [PubMed: 23749645]

12. Hankin JH, Stram DO, Arakawa K, Park S, Low SH, Lee HP, et al. Singapore Chinese Health Study: development, validation, and calibration of the quantitative food frequency questionnaire. Nutr Cancer. 2001; 39:187-95. [PubMed: 11759279]

13. Parkin, DM.; Whelan, SL.; Ferlay, J.; Teppo, L.; Thomas, D. Cancer Incidence in Five Continents. Lyon: IARC; 2002. 
14. Koh WP, Robien K, Wang R, Govindarajan S, Yuan JM, Yu MC. Smoking as an independent risk factor for hepatocellular carcinoma: the Singapore Chinese Health Study. Br J Cancer. 2011; 105:1430-5. [PubMed: 21915129]

15. Willett WC, Howe GR, Kushi LH. Adjustment for total energy intake in epidemiologic studies. Am J Clin Nutr. 1997; 65:1220S-28S. discussion 29S-31S. [PubMed: 9094926]

16. WHO expert consultation. Appropriate body-mass index for Asian populations and its implications for policy and intervention strategies. Lancet. 2004; 363:157-63. [PubMed: 14726171]

17. Patterson E, Wall R, Fitzgerald GF, Ross RP, Stanton C. Health implications of high dietary omega-6 polyunsaturated Fatty acids. J Nutr Metab. 2012; 2012:539426. [PubMed: 22570770]

18. Di Minno MN, Russolillo A, Lupoli R, Ambrosino P, Di Minno A, Tarantino G. Omega-3 fatty acids for the treatment of non-alcoholic fatty liver disease. World J Gastroenterol. 2012; 18:583947. [PubMed: 23139599]

19. Jump DB. N-3 polyunsaturated fatty acid regulation of hepatic gene transcription. Curr Opin Lipidol. 2008; 19:242-7. [PubMed: 18460914]

20. Calder PC. Polyunsaturated fatty acids and inflammation. Prostaglandins Leukot Essent Fatty Acids. 2006; 75:197-202. [PubMed: 16828270]

21. Monteiro J, Leslie M, Moghadasian MH, Arendt BM, Allard JP, Ma DW. The role of $\mathrm{n}-6$ and n 3 polyunsaturated fatty acids in the manifestation of the metabolic syndrome in cardiovascular disease and non-alcoholic fatty liver disease. Food Funct. 2014; 5:426-35. [PubMed: 24496399]

22. Hashimoto E, Tokushige K. Hepatocellular carcinoma in non-alcoholic steatohepatitis: Growing evidence of an epidemic? Hepatol Res. 2012; 42:1-14. [PubMed: 21917086]

23. Page JM, Harrison SA. NASH and HCC. Clin Liver Dis. 2009; 13:631-47. [PubMed: 19818310]

24. Puri P, Baillie RA, Wiest MM, Mirshahi F, Choudhury J, Cheung O, et al. A lipidomic analysis of nonalcoholic fatty liver disease. Hepatology. 2007; 46:1081-90. [PubMed: 17654743]

25. Cortez-Pinto H, Jesus L, Barros H, Lopes C, Moura MC, Camilo ME. How different is the dietary pattern in non-alcoholic steatohepatitis patients? Clin Nutr. 2006; 25:816-23. [PubMed: 16677739]

26. Poonawala A, Nair SP, Thuluvath PJ. Prevalence of obesity and diabetes in patients with cryptogenic cirrhosis: a case-control study. Hepatology. 2000; 32:689-92. [PubMed: 11003611]

27. Koh WP, Yuan JM, Sun CL, Lee HP, Yu MC. Middle-aged and older chinese men and women in singapore who smoke have less healthy diets and lifestyles than nonsmokers. J Nutr. 2005; 135:2473-7. [PubMed: 16177215]

28. El-Serag HB, Tran T, Everhart JE. Diabetes increases the risk of chronic liver disease and hepatocellular carcinoma. Gastroenterology. 2004; 126:460-8. [PubMed: 14762783]

29. Younossi ZM, Gramlich T, Matteoni CA, Boparai N, Mccullough AJ. Nonalcoholic fatty liver disease in patients with type 2 diabetes. Clin Gastroenterol Hepatol. 2004; 2:262-5. [PubMed: 15017611]

30. Diehl AM. Hepatic complications of obesity. Gastroenterol Clin North Am. 2010; 39:57-68. [PubMed: 20202579]

31. Vernon G, Baranova A, Younossi ZM. Systematic review: the epidemiology and natural history of non-alcoholic fatty liver disease and non-alcoholic steatohepatitis in adults. Aliment Pharmacol Ther. 2011; 34:274-85. [PubMed: 21623852]

32. Kremsdorf D, Soussan P, Paterlini-Brechot P, Brechot C. Hepatitis B virus-related hepatocellular carcinoma: paradigms for viral-related human carcinogenesis. Oncogene. 2006; 25:3823-33. [PubMed: 16799624]

33. Anzola M. Hepatocellular carcinoma: role of hepatitis B and hepatitis C viruses proteins in hepatocarcinogenesis. J Viral Hepat. 2004; 11:383-93. [PubMed: 15357643]

34. Kuper H, Tzonou A, Lagiou P, Mucci LA, Trichopoulos D, Stuver SO, et al. Diet and hepatocellular carcinoma: a case-control study in Greece. Nutr Cancer. 2000; 38:6-12. [PubMed: 11341045]

35. Polesel J, Talamini R, Montella M, Maso LD, Crovatto M, Parpinel M, et al. Nutrients intake and the risk of hepatocellular carcinoma in Italy. Eur J Cancer. 2007; 43:2381-7. [PubMed: 17719221] 
36. Koh WP, Yang HN, Yang HQ, Low SH, Seow A. Potential sources of carcinogenic heterocyclic amines in the Chinese diet: results from a 24-h dietary recall study in Singapore. Eur J Clin Nutr. 2005; 59:16-23. [PubMed: 15329677]

\section{List of abbreviations}

$\begin{array}{ll}\text { PUFA } & \text { polyunsaturated fatty acids } \\ \text { HCC } & \text { hepatocellular carcinoma } \\ \text { NASH } & \text { non-alcoholic steatohepatitis } \\ \text { HBV } & \text { hepatitis B virus } \\ \text { HCV } & \text { hepatitis C virus } \\ \text { HR } & \text { hazard ratio } \\ \text { CI } & \text { confidence interval }\end{array}$




\section{Key points}

1. Recent work on the lipidomic signature of lipid metabolism suggests that long chain omega-6 polyunsaturated fatty acids (PUFA) may play a role in hepatocarcinogenesis.

2. Our study is the first to show that the intake of omega-6 PUFA was associated with increased risk of hepatocellular carcinoma (HCC).

3. These results provided the first epidemiologic evidence for the role of dietary omega-6 PUFA in the development of HCC, possibly by rendering chronic inflammation in non-alcoholic steatohepatitis. 
Table 1

Participant characteristics [n (\%) or mean \pm standard deviation] according to quartiles of omega-6 PUFA intake

\begin{tabular}{lcccc}
\hline & \multicolumn{4}{c}{ Quartiles of total omega-6 PUFA of total omega 6 PUFA } \\
\hline & Q1 & Q2 & Q3 & Q4 \\
\hline Omega-6 PUFA (\% kcal) (interquartile range) & $2.4-3.1$ & $3.5-4.0$ & $4.4-5.0$ & $5.9-7.7$ \\
\hline Number of participants & 15033 & 15142 & 15094 & 15029 \\
Age at recruitment, years & $57.6 \pm 8.0$ & $56.5 \pm 8.0$ & $55.9 \pm 8.0$ & $55.5 \pm 7.8$ \\
Female sex, n (\%) & $7155(47.6)$ & $8200(54.2)$ & $8709(57.7)$ & $9400(62.6)$ \\
Cantonese Dialect, n (\%) & $6252(41.6)$ & $6715(44.4)$ & $7283(48.3)$ & $7673(51.1)$ \\
Education, secondary school or higher, n (\%) & $2962(19.7)$ & $3903(25.8)$ & $4689(31.1)$ & $5626(37.4)$ \\
Ever smoker, $\mathrm{n}(\%)$ & $5940(39.5)$ & $4847(32.0)$ & $4212(27.9)$ & $3389(22.6)$ \\
Weekly/daily alcohol drinker, $\mathrm{n}(\%)$ & $2367(15.8)$ & $1785(11.8)$ & $1586(10.5)$ & $1271(8.5)$ \\
Coffee drinkers of at least 2 cups/day, $\mathrm{n}(\%)$ & $5713(38.0)$ & $5449(36.0)$ & $4996(33.1)$ & $4564(30.4)$ \\
Diabetes at recruitment, $\mathrm{n}(\%)$ & $1131(7.5)$ & $1298(8.6)$ & $1396(9.3)$ & $1528(10.2)$ \\
Body mass index, kg/m ${ }^{2}$ & $23.1 \pm 3.2$ & $23.1 \pm 3.3$ & $23.1 \pm 3.3$ & $23.2 \pm 3.3$ \\
Total energy, kcal/d & $1487 \pm 507$ & $1547 \pm 520$ & $1597 \pm 532$ & $1561 \pm 515$ \\
Total protein (\% kcal/d) & $13.6 \pm 2.2)$ & $15.2 \pm 2.2$ & $15.8 \pm 2.3$ & $16.2 \pm 2.3$ \\
Total fat (\% kcal/d) & $19.9 \pm 4.2$ & $25.0 \pm 4.2$ & $27.2 \pm 5.1$ & $28.3 \pm 4.8$ \\
Saturated fatty acids (\% kcal/d) & $7.9 \pm 2.2$ & $9.5 \pm 2.3$ & $9.6 \pm 2.8$ & $8.4 \pm 2.3$ \\
Monounsaturated fatty acids (\% kcal/d) & $6.7 \pm 1.5$ & $8.6 \pm 1.5$ & $9.4 \pm 1.9$ & $9.2 \pm 2.1$ \\
Omega-3 PUFA (\% kcal/d) & $0.40 \pm 0.10$ & $0.49 \pm 0.10$ & $0.54 \pm 0.12$ & $0.64 \pm 0.23$ \\
\hline
\end{tabular}

Chi-square test was used for categorical variables; one-way ANOVA was used for continuous variables; all p-values $<0.001$ 
Table 2

Participant characteristics [n (\%) or mean \pm standard deviation] according to hepatocellular carcinoma status

\begin{tabular}{lccc}
\hline & Hepato cellular carcinoma cases & Non-cases & P value \\
\hline Number of participants & 488 & 59810 & \\
Age, y & $60.4 \pm 7.3$ & $56.4 \pm 8.0$ & $<0.001$ \\
Female sex, n (\%) & $134(27.5)$ & $33300(55.7)$ & $<0.001$ \\
Cantonese Dialect, n (\%) & $188(38.5)$ & $27735(46.4)$ & $<0.001$ \\
Education, secondary school or higher, n (\%) & $113(23.2)$ & $17067(28.5)$ & 0.009 \\
Ever smoker, n (\%) & $268(54.9)$ & $18120(30.3)$ & $<0.001$ \\
Weekly/daily alcohol drinker, n (\%) & $87(17.8)$ & $6922(11.6)$ & $<0.001$ \\
Coffee drinkers of at least 2 cups/day, n (\%) & $151(30.9)$ & $20571(34.4)$ & 0.11 \\
Diabetes, $\mathrm{n}(\%)$ & $88(18.0)$ & $5265(8.8)$ & $<0.001$ \\
Body mass index, kg/m ${ }^{2}$ & $23.9 \pm 3.4$ & $23.1 \pm 3.3$ & $<0.001$ \\
Total energy, kcal/d & $1553.9 \pm 539.8$ & $1548.0 \pm 519.8$ & 0.80 \\
Total protein (\% kcal/d) & $15.1 \pm 2.5$ & $15.2 \pm 2.5$ & 0.53 \\
Total fat (\% kcal/d) & $24.6 \pm 6.0$ & $25.1 \pm 5.6$ & 0.08 \\
Saturated fatty acids (\% kcal/d) & $8.6 \pm 2.5$ & $8.9 \pm 2.5$ & 0.04 \\
Monounsaturated fatty acids (\% kcal/d) & $8.4 \pm 2.0$ & $8.5 \pm 2.1$ & 0.25 \\
Omega-3 PUFA (\% kcal/d) & $0.51 \pm 0.17$ & $0.52 \pm 0.17$ & 0.32 \\
\hline
\end{tabular}

Chi-square test was used for categorical variables; one-way ANOVA was used for continuous variables 


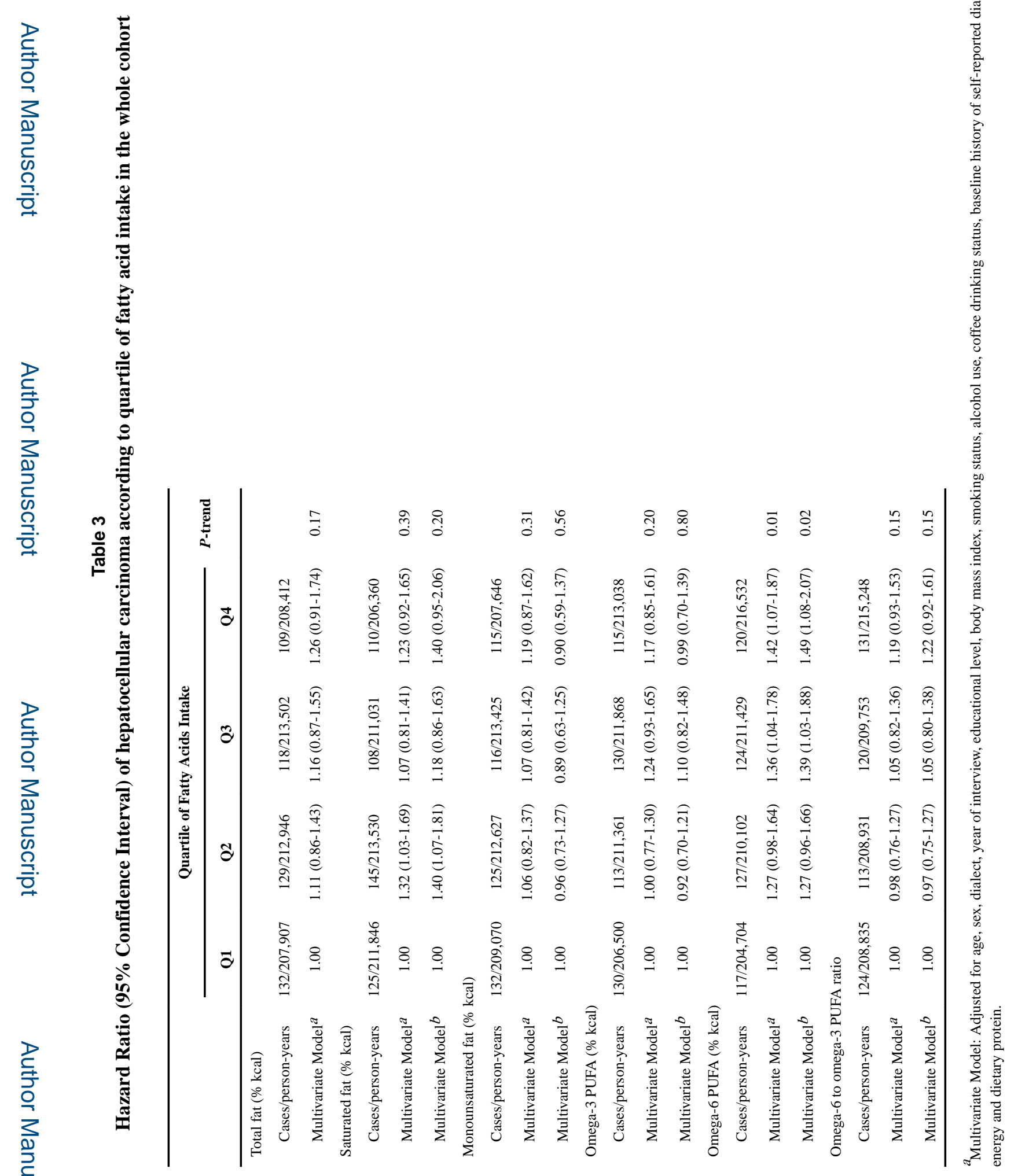




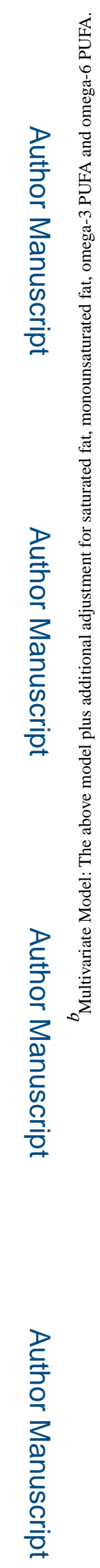


Table 4

Odds Ratio (95\% Confidence Interval) of hepatocellular carcinoma according to fatty acids intake in a case-control study nested within the cohort

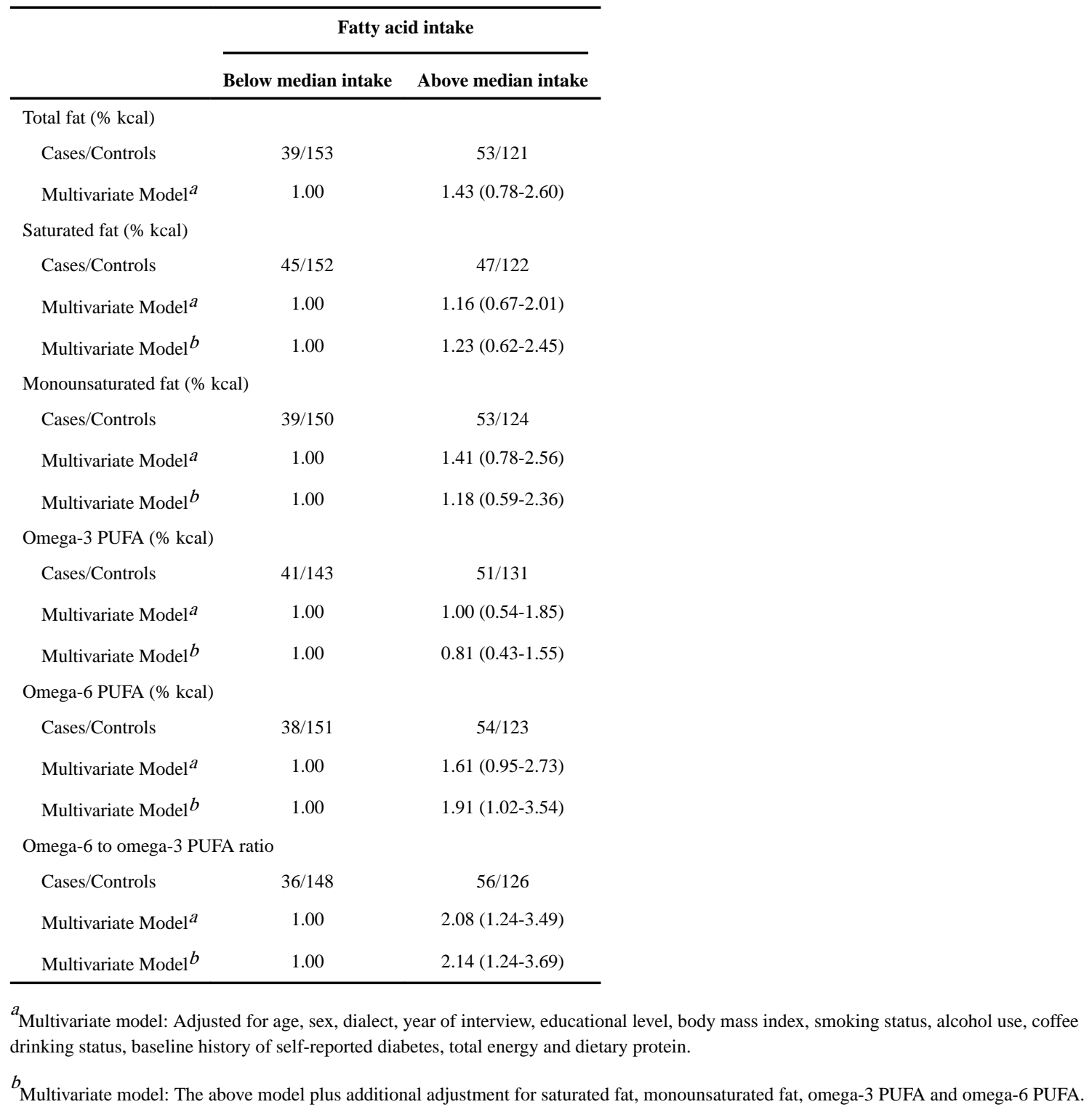




\section{Table 5}

Odds Ratio (95\% Confidence Interval) of hepatocellular carcinoma according to fatty acids intake in a casecontrol study nested within the cohort.

\begin{tabular}{|c|c|c|c|c|}
\hline & \multicolumn{2}{|c|}{ Subjects negative for $\mathrm{HBV}$ and $\mathrm{HCV}^{c}$} & \multicolumn{2}{|c|}{ Subjects positive for $\mathrm{HBV}$ or $\mathrm{HCV}^{c}$} \\
\hline & Below the median & Above the median & Below the median & Above the median \\
\hline \multicolumn{5}{|l|}{ Omega-3 PUFA (\% kcal) } \\
\hline Cases/Controls & $17 / 115$ & $21 / 106$ & $24 / 28$ & $30 / 25$ \\
\hline Multivariate Model $^{a}$ & 1.00 & $1.02(0.38-2.73)$ & 1.00 & $0.95(0.31-2.88)$ \\
\hline Multivariate Model $b$ & 1.00 & $0.71(0.25-2.05)$ & 1.00 & $0.69(0.20-2.35)$ \\
\hline \multicolumn{5}{|l|}{ Omega-6 PUFA ( $\%$ kcal) } \\
\hline Cases/Controls & $14 / 127$ & $24 / 94$ & $24 / 24$ & $30 / 29$ \\
\hline Multivariate Model $^{a}$ & 1.00 & $2.87(1.25-6.58)$ & 1.00 & $0.65(0.25-1.73)$ \\
\hline Multivariate Model $b$ & 1.00 & $4.36(1.59-11.94)$ & 1.00 & $0.81(0.25-2.65)$ \\
\hline \multicolumn{5}{|c|}{ Omega- 6 to omega-3 PUFA ratio } \\
\hline Cases/Controls & $13 / 123$ & $25 / 98$ & $23 / 25$ & $31 / 28$ \\
\hline Multivariate Model ${ }^{a}$ & 1.00 & $2.86(1.28-6.39)$ & 1.00 & $1.12(0.45-2.79)$ \\
\hline Multivariate Model $b$ & 1.00 & $2.98(1.26-7.05)$ & 1.00 & $1.27(0.48-3.39)$ \\
\hline
\end{tabular}

